\title{
La industria de la música popular en España: los sellos independientes en la era digital
}

\section{Luis A. Albornoz y J. Ignacio Gallego}

\section{Resumen}

El artículo presenta los resultados de un estudio realizado con discográficas y distribuidoras independientes que actúan en el mercado de la música popular en España. Con la finalidad de obtener una suerte de fotografía de la situación de este sector y analizar sus tendencias, se administró una encuesta online durante el primer semestre de 2011 a este tipo de empresas. El estudio dirigido por investigadores del Departamento de Periodismo y Comunicación Audiovisual de la Universidad Carlos III de Madrid, contó con el apoyo de la Unión Fonográfica Independiente. El artículo incide, principalmente, en las diversas actividades y estrategias que desarrollan los sellos y distribuidoras independientes, las cuales incluyen una activa presencia en las nuevas redes y plataformas digitales. Los resultados de la investigación se presentan en el siguiente orden: a) perfil de las empresas, b) presencia internacional, c) promoción y publicidad, y d) internet y servicios online.

\section{Palabras clave}

Industria musical. Discográficas independientes. Internet. Música popular. España.

Luis A. Albornoz I luisalfonso.albornoz@uc3m.es Doctor en Comunicación por la Universidad Complutense de Madrid (UCM). Profesor de los programas de Grado y Postgrado de la Universidad Carlos III de Madrid (UC3M).

J. Ignacio Gallego I juanignacio.gallego@uc3m.es Doctor en Comunicación por la Universidad Complutense de Madrid (UCM). Profesor de los programas de Grado de la Universidad Carlos III de Madrid (UC3M).

\section{Introducción}

El presente artículo se acerca a la industria fonográfica de música popular1 a través del análisis de un sector de estimable relevancia dentro de la producción cultural de España: el de las llamadas empresas independientes. Entendiendo que es un sector conformado por una pluralidad de agentes-compositores e intérpretes, sellos discográficos, compañías distribuidoras, promotores, agentes de artistas, representantes de festivales y otros profesionales y amateurs- que operan en el mercado musical complementando la actuación de los principales conglomerados mediáticos internacionales y locales (ALBORNOZ; GALLEG0, 2011, p. 87).

En España las empresas independientes son actualmente las mayores productoras y distribuidoras de fonogramas, al ser las responsables, junto a los artistas que autoeditan sus trabajos, del 80\% de las novedades musicales que se lanzan al mercado (UFI, 2010). Sin embargo, las firmas independientes tienen un 
peso marginal en el mercado local ya que éste, altamente transnacionalizado, está dominado por las majors (Sony Music, Universal Music, WarnerDro y EMI Music) las cuales generan los mayores volúmenes de ventas de fonogramas. ${ }^{2}$

Por otra parte, las estadísticas oficiales acerca del mercado español de fonogramas suelen desconocer la actuación de las empresas independientes.

Por ejemplo, los datos que anualmente reporta la patronal del sector, Productores de Música de España - Promusicae, se basan en la información facilitada por las majors y otras diez empresas: Avispa, Blanco y Negro, Boa, Dial, Discmedi, Divucsa, Emi Music, Harmonia Mundi Ibérica, Naïve y Open. Por tanto, no tiene en consideración las actividades comerciales de un importante número de sellos y distribuidoras -se calcula que existe un centenar de firmas independientes en activo- que forman parte del entramado musical español. Por otro lado, el informe periódico de la entidad a cargo de la gestión de derechos de autor de compositores e intérpretes, la Sociedad General de Autores y Editores (SGAE), en su capítulo dedicado a la música grabada, se limita a ofrecer una serie de datos generales sobre los sellos independientes sin facilitar su volumen de mercado.
En este marco, durante el primer semestre de 2011 investigadores del Departamento de Periodismo y Comunicación Audiovisual de la Universidad Carlos III de Madrid (UC3M) realizaron una investigación con la finalidad de conocer la situación en que se encuentran las empresas independientes que actúan en la comercialización de grabaciones de música, indagando en su relación con el tejido mediático tradicional y nuevo, su aportación a la diversidad musical y su capacidad de convertirse en un sector cultural relevante a nivel nacional e internacional.

\section{Sellos y distribuidoras independientes en España}

La aplicación de la etiqueta independiente 0 indie (abreviatura en lengua inglesa de la palabra independent) a un determinado sector de la industria 0 a un determinado género musical ha sido (y es) fuente de confusión y debate al ser abordada desde diferentes perspectivas. Por un lado, la definición industrial, que aborda el análisis de la estructura y funcionamiento del mercado de la música, entiende que el sector independiente en el terreno musical está conformado por aquellas empresas, generalmente

Como sostiene Shuker (2005, p. 214): "Esencialmente, toda la música popular consiste en un híbrido de tradiciones, estilos e influencias musicales, y también es un producto económico al que muchos de sus consumidores invierten de un significado ideológico. En el núcleo de la mayoría de los diversos tipos de música popular hay una tensión fundamental entre la creatividad esencial del acto de 'hacer música' y la naturaleza comercial del grueso de su producción y difusión".

En 2011 la participación de las majors alcanzó el 88,22\% del mercado fonográfico en soporte físico -Universal: 29,06\%, Sony: 25,11\%, Warner: 18,66\% y EMl: 15,39\%- y el 96,92\% del mercado digital -Universal: 38,05\%, Sony: 25,78\%, Warner: 18,07\% y EMI: 15,02\%- (PROMUSICAE, 2012a y 2012b). 
de talla pequeña, que se encargan de la

producción y distribución de fonogramas y de la promoción de artistas por fuera de las grandes corporaciones multinacionales. Por otro lado, para el enfoque estético-político lo independiente representa una concepción contracultural que vincula la producción sonora resultante con una determinada posición institucional y política al margen de las corporaciones en la búsqueda de la diversidad sonora. En este sentido, según Hesmondhalgh, el indie se convirtió durante la década de 1980 en el primer género musical que tomó su nombre a partir de la forma de organización industrial que lo sustentaba (HESMONDHALGH, 1999, p. 35-36). Autoras como Fonarow (2006) o Kruse (2003), por su parte, recurren al término autenticidad y al enfrentamiento industrial/estético que la música independiente plantea a la corriente dominante 0 mainstream, conformado por las grandes firmas comerciales y su start system.

El desarrollo de la etiqueta independiente en el ámbito de la música popular (como forma de diferenciación y de producto) se ha producido en la industria española en las últimas tres décadas. Los sellos independientes vienen cumpliendo en los últimos años un papel clave a la hora de descubrir y apostar por nuevos artistas y tendencias de musicales. A su vez estos sellos, a través de sus distintas actividades, vienen complementando la actuación de las majors discográficas en una estructura de mercado controlada por éstas últimas. Como señala Buquet:
Las propias empresas multinacionales consideran la labor de los sellos pequeños como parte importante de la maquinaria de la industria fonográfica, que consiste en producir un disco de un grupo que no se conoce fuera de su reducido circuito, situarlo en el mercado, obtener beneficios, vender el grupo a una multinacional y seguir en la búsqueda de nuevos talentos (BUQUET, 2002, p. 73).

En España el sector de empresas independientes da sus primeros pasos durante la década de 1980, cuando surgen los primeros sellos que ganarían reputación. Discos Radioactivos Organizados (DR0), Grabaciones Accidentales (GASA), Twins, 3 Cipreses, Nuevos Medios o Lollipop son pioneros en comenzar a desarrollar un sector independiente y alternativo del dominado por las grandes discográficas nacionales y multinacionales. El Grupo DR0 (que agrupaba a las discográficas DR0, Twins y GASA) alcanzó tal nivel de éxito que fue absorbido por la multinacional Warner en 1993.

En la década de 1990 surge una nueva hornada de sellos vinculada directamente a la música indie y a la emergencia del hip hop con nombres como Acuarela, Elefant, Subterfuge o BOA. A este conjunto destacado de casas discográficas hay que añadir aquellas que, aun siendo independientes de las grandes, alcanzan una mayor cuota de mercado y compiten en nichos de mercado específicos. Destacan entre éstas: Discmedi (pop en catalán, 3,73\% del mercado), Harmonia mundi (multinacional de origen francés especializada en música culta, aunque también distribuye 
jazz, world music o pop-rock; 1,16\%), Divucsa

(recopilatorios y maxis de música dance, 1,11\%) y

Avispa Music (especializado en heavy metal, 1,04\%)

(SGAE, 2012).

Por último, se debe destacar la labor de las empresas distribuidoras que se encargan de colocar en el mercado una cantidad importante de novedades extranjeras dotando al mercado de una importante diversidad con lanzamientos de todo tipo de sellos que abarcan una importante cantidad de estilos. Popstock, Pias, Green Ufos, Karonte, Music As Usual o Discmedi son algunas de estas distribuidoras que además suelen dedicarse, de forma intermitente, a la producción.

En noviembre de 2003, cerca de medio centenar de productoras, distribuidoras y promotoras (en la actualidad son más de 60 empresas) decidieron aglutinarse en torno a la Unión Fonográfica Independiente (UFI) con la finalidad de:

reivindicar y mejorar las posibilidades de acceso al mercado de un sector que tradicionalmente ha sido el motor creativo de la industria discográfica, por la capacidad de reinventarse y por la enorme contribución al patrimonio musical de nuestro país (UFI, 2012).

En su carta estatutaria la UFI establece que pueden integrar la asociación cualquiera que se dedique a la producción fonográfica "de modo independiente" y que no ostente un porcentaje del mercado fonográfico español superior al 5\%. La condición de dedicarse "de modo independiente" está determinada por el cumplimiento de las siguientes condiciones: no estar integrado en grupos empresariales que desempeñen actividades ajenas al sector musical, no estar participado ni controlado por medios de comunicación y no estar vinculado a entidades de gestión colectiva de derechos de propiedad intelectual, salvo en calidad de mero asociado beneficiario de sus servicios (ALBORNOZ, 2011b).

Ante este panorama, el sector de empresas independientes que actúa en el mercado de la música popular en España debe sopesar su importancia tomando en consideración las siguientes cuestiones:

Aporte a la diversidad musical: la apuesta por estilos musicales, artistas o lenguas tanto nacionales como internacionales que quedan fuera de los lanzamientos discográficos de las majors, hace que los agentes del sector independiente sean fundamentales para el desarrollo de una industria cultural que apueste diversa. Se convierten así en piezas claves, tanto en la exportación de cultura española como en la importación de sonidos creados y producidos en otras geografías.

Lanzamiento de nuevos artistas: la apuesta por artistas nóveles que sólo encuentran una salida a su trabajo creativo en este tipo de sellos. No sólo se trata de ofrecer servicios de producción (pues muchos artistas llegan con su trabajo musical ya autoeditado), si no de ofrecer múltiples servicios que van desde la promoción en medios de comunicación especializados y generalistas, a la gestión del merchandising, incluyendo la contratación para actuar en conciertos y festivales.

Creación de un tejido industrial local: ante la transnacionalización de las grandes compañí- 
as y el progresivo proceso de concentración que les afecta, es fundamental el papel de las micro y pequeñas empresas independientes a la hora de generar una industria que esté anclada a lo local.

\section{Metodología}

Teniendo en consideración la importancia de las empresas independientes en el mercado de la música popular en España y la falta de estudios que evalúen la realidad de este sector, se tomó la decisión de realizar la encuesta titulada Situación de la industria musical independiente en España, 2011. El objetivo del estudio, que contó con el apoyo de la UFI, fue realizar una fotografía de la situación actual de las compañías discográficas y distribuidoras independientes.

Con esta finalidad se diseño una encuesta que se administró entre febrero y mayo de 2011 a través de SurveyMonkey, plataforma especializada en encuestas a través de internet. El cuestionario fue enviado a 83 empresas del sector (sellos discográficos, principalmente, y algunas distribuidoras): 60 de éstas pertenecientes a la UFI y 23 no afiliadas a esta asociación (APÊNDICE A). La encuesta se complementó con un seguimiento a través de llamados telefónicos.

La encuesta se dividió en las siguientes tres secciones:

a) Datos básicos de la empresa: Razón social. Fecha de inicio de actividades.
Estilos musicales con los trabaja.

Pertenencia a asociaciones y

entidades de gestión.

Número de empleados.

Facturación anual.

b) Actividades desarrolladas:

Tipo de actividades y

porcentajes de facturación.

Artistas en catálogo.

Formatos de publicación

Licencias de publicación.

Presencia en ferias internacionales

Subvenciones recibidas.

c) Medios de comunicación, promoción

y redes digitales:

Inversión en publicidad.

Mecanismos de promoción.

Presencia en redes sociales e internet.

Presencia en servicios de distribución de música online.

Relación con agregadores musicales.

\section{Principales resultados}

Antes de presentar los principales resultados, es preciso realizar unos comentarios acerca de las dificultades que enfrentó la realización de este estudio. A pesar de que el mismo contó con el apoyo institucional de la UFI y se procuró explicar su finalidad entre empresarios, un número importante de firmas no respondió la encuesta 0 lo hizo sólo parcialmente. Esto obligó a insistir en la importancia de responder la encuesta a través de mensajes electrónicos y llamadas telefónicas, y a mantener la plataforma online con la encuesta abierta mayor tiempo del previsto.

A pesar que muchas de las discográficas y distribuidoras contactadas están obligadas a declarar su facturación anual ante el Registro 
Mercantil (entidad administrativa que tiene por objeto principal la publicidad oficial de las situaciones jurídicas de los empresarios en él inscritos), el hecho de que el cuestionario elaborado indagara en los resultados económicos de las firmas (se preguntó por los ingresos correspondientes a los años 2000,2005 y 2010) fue, sin duda, un elemento disuasorio para muchos empresarios. Por tanto, el objetivo de conocer el peso económico de este sector no pudo ser alcanzado. Esta lamentable falta de transparencia, a nuestro entender, atenta contra el conocimiento de un sector clave en la industria de la música española actual.

Por otra parte, se constató que la mayoría de los sellos independientes son microempresas con una escaza reacción de respuesta. Cuentan con muy pocos responsables/trabajadores los cuales deben cumplir múltiples funciones: desde la producción de grabaciones sonoras hasta la promoción de artistas, pasando por la organización de conciertos en vivo. Estas microempresas multiservicios, por tanto, tienen una baja capacidad de reacción frente a la demanda de información externa. Resulta evidente que responder los requerimientos de un estudio para conocer la realidad del sector de la música independiente no forma parte de sus prioridades del día a día.

En resumen, la encuesta online fue respondida de forma desigual por 45 empresas, con algunas preguntas que no fueron contestadas o que no eran útiles al no tener un volumen suficiente de respuestas para poder ofrecer un dato contrastado. Esto no impidió la obtención de valiosos datos para conocer y analizar el actual funcionamiento de las empresas independientes en el terreno de la música popular y sus relaciones con el sector de los medios de comunicación y las redes digitales.

\section{Perfil de empresas}

La mayoría de las empresas encuestadas comenzaron sus actividades en Madrid y Barcelona durante la década de 2000 (27 empresas) frente a las que lo hicieron en el periodo comprendido entre los años 1989 y 1999 (13 empresas), con un claro repunte de inicio de actividades en 2010 en el que se pusieron en marcha cinco de los sellos encuestados.

Desde el punto de vista del número de trabajadores que estas empresas tienen contratado cabe destacar que la mayoría son microempresas ${ }^{3}$ que actualmente emplean entre

En la Unión Europea las microempresas y las pequeñas y medianas empresas se definen en función de sus efectivos y de su volumen de negocios o de su balance general anual. Se define a una mediana empresa como una empresa que ocupa a menos de 250 personas y cuyo volumen de negocios anual no excede de 50 millones de euros 0 cuyo balance general anual no excede de 43 millones de euros; a una pequeña empresa como una empresa que ocupa a menos de 50 personas y cuyo volumen de negocios anual o cuyo balance general anual no supera los 10 millones de euros; a una microempresa como una empresa que ocupa a menos de 10 personas y cuyo volumen de negocios anual o cuyo balance general anual no supera los 2 millones de euros. 
uno y tres trabajadores $(62 \%$ de las empresas encuestadas), entre 5 y 6 ( $23 \%$ ), entre 7 y 10 (11\%). Hoy en día, solamente una empresa dedicada a la distribución de trabajos musicales emplea más de diez trabajadores y puede ser considerada, según esta variable, como una pequeña empresa.

Complementariamente, a fin de elaborar los perfiles de los sellos independientes se preguntó por el tipo de estilos musicales con los que trabajan, tomando en consideración la clasificación de once categorías artísticas musicales realizada por la UFI para la $3^{\text {a }}$ edición de los Premios de la música independiente, 2011. Una amplia mayoría de las empresas encuestadas trabaja con Pop (más del 80\%) y Rock (más del 70\%), estilos seguidos por la música Electrónica (50\% aprox.).
En relación con el tipo de actividades que realizan las discográficas y distribuidoras independientes, se comprueba que además de la producción fonográfica, actividad desarrollada por la totalidad de las empresas encuestadas, la gran mayoría de éstas se dedica a otras diferentes tareas. Entre éstas se destacan la gestión de los derechos de explotación comercial de la obra de sus artistas y de otros a través de una sección Editorial (75\% aprox.), la gestión de las carreras artísticas de sus propios músicos a través de la figura del manager (70\% aprox.) y la venta de grabaciones al público a través de tiendas en internet (60\% aprox.).

Por otra parte, ante la transformación de los formatos de publicación de grabaciones y la eclosión de un mercado de archivos digitales que convive con el resurgimiento de los discos de vinilo,

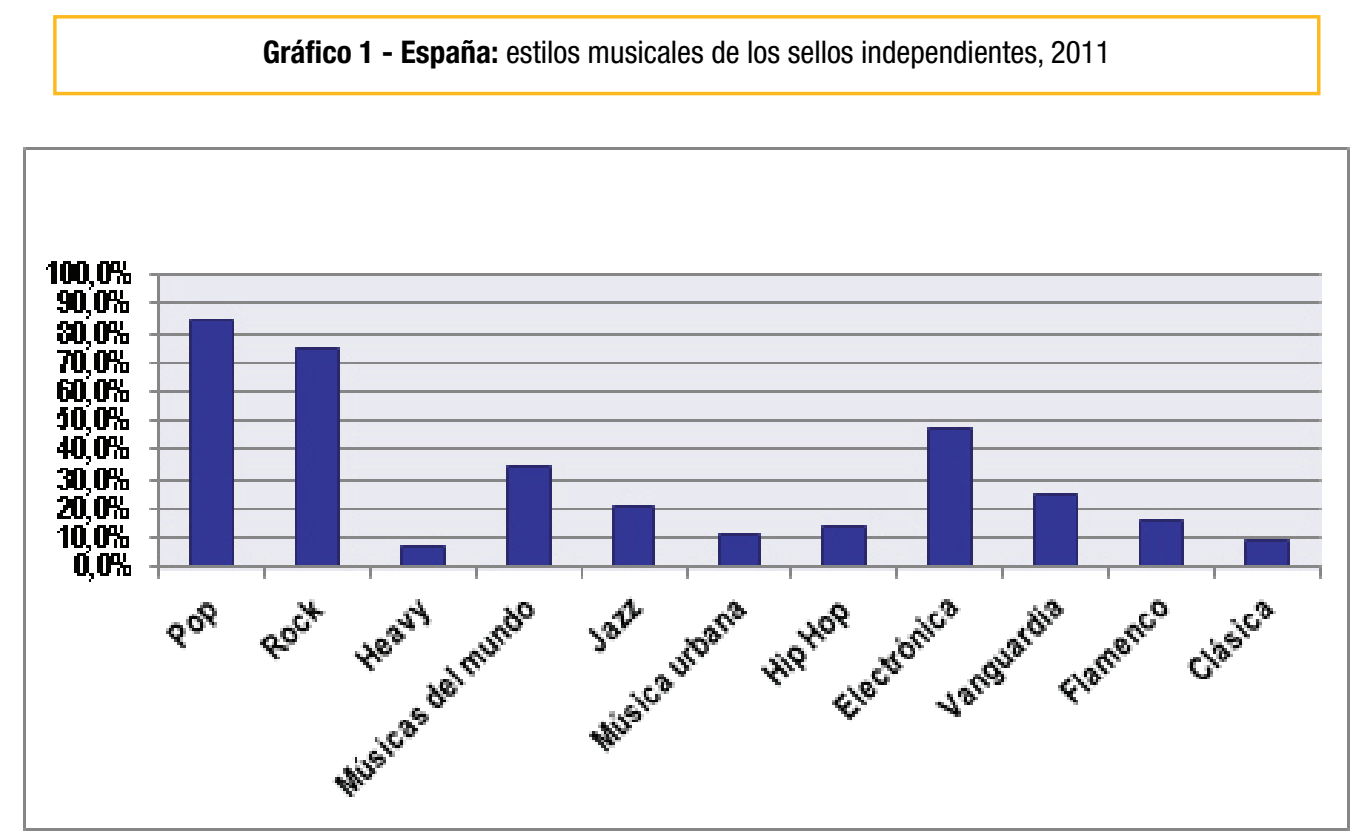

Fuente: elaboración propia. 
se indagó acerca de los tipos de formatos con el que trabajaban los sellos independientes españoles.

En este aspecto se destaca que la totalidad de las discográficas consultadas continúa publicando obras en soporte compact disk (CD) debido a su alta rentabilidad frente a la de otros formatos y, como se verá más adelante, a que este formato es ideal para promocionar artistas y trabajos entre los periodistas del sector. Asimismo el long play (LP) en soporte vinilo se destaca por ser un "viejo" formato que hoy está siendo utilizado por más del $60 \%$ de los sellos, ganándole a la publicación de grabaciones en Mp3 (56\%).

Además de los diferentes formatos con los que trabajan, se preguntó a las empresas independientes por el tipo de licencias que utilizan de cara a la circulación de las obras musicales. Si bien la gran mayoría sigue apostando por el copyright (91\% sobre 34 respuestas recibidas), seis sellos respondieron (17\%) estar utilizando licencias Creative Commons para la publicación de referencias musicales, las cuales permitir usos no comerciales de las mismas (véase: http:// es.creativecommons.org/licencia/). En este sentido, la decisión de emplear licencias Creative Commons no depende únicamente del sello discográfico, sino que tanto artistas como autores deben estar de acuerdo en la forma de licenciar sus creaciones.

\section{Presencia internacional}

Otra de las cuestiones planteadas en el estudio fue la capacidad de las empresas independientes de tener presencia en mercados foráneos. En consecuencia se preguntó sobre el licenciamiento

Gráfico 2 - España: actividades de las discográficas y distribuidoras independientes, 2011

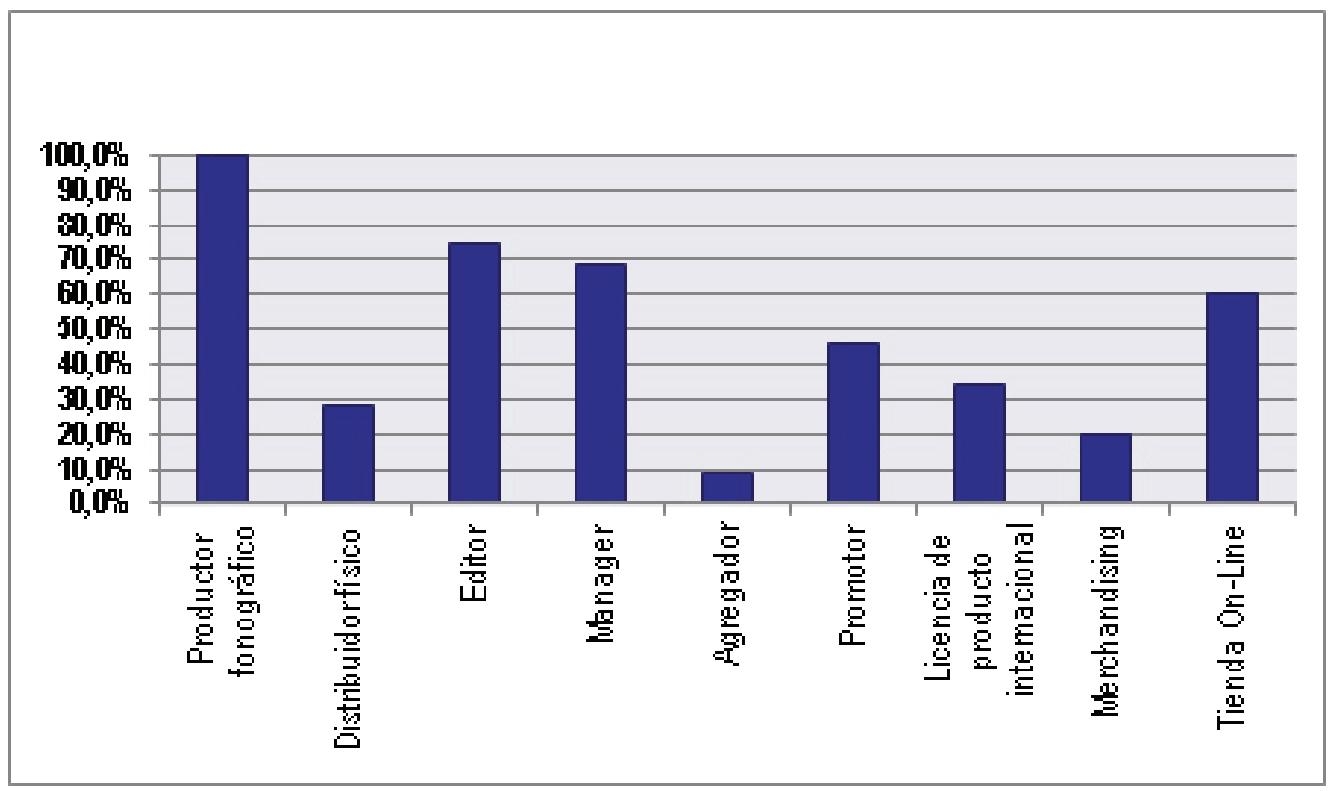

Fuente: elaboración propia. 
de obras musicales, la exportación y venta de grabaciones a otros países y la presencia de firmas independientes en las ferias de negocios internacionales. Así, poco más de la mitad de las empresas que respondieron (un 53\% sobre un total de 32 respuestas recibidas) manifestó licenciar 0 distribuir material en diferentes mercados extranjeros. Entre estos últimos se destacan, por este orden, los países miembros de la Unión Europea -especialmente Reino Unido, Francia y Alemania- Estados Unidos, Japón y México.

Respecto a la presencia de empresas independientes en ferias internacionales, se destaca la asistencia a tres eventos principales: el Marché international du disque et de l'édition musicale - Midem, realizado anualmente en Cannes, Francia; el The World Music Expo Womex, originario de Berlín, Alemania, y que actualmente se celebra en diferentes localidades de Europa; y el South by West - SXSW, de Austin, Estados Unidos. La presencia de las empresas independientes españolas en este tipo de importantes ferias internacionales en algunos casos cuenta con el apoyo y la ayuda económica de instituciones públicas como el Instituto Español de Comercio Exterior (ICEX), a través de su programa de internacionalización de la música producida en el país, denominado Sounds from Spain (SfS).

\section{El ICEX, al igual que otras asociaciones 0} instituciones, forma parte de la batería de benefactores de los sellos y distribuidoras independientes. Aproximadamente un 46\% (sobre 35 respuestas recibidas) de las empresas afirmó haber recibido algún tipo de ayuda o subvención para la producción o promoción de sus catálogos. Al margen del ICEX se destacan las ayudas dadas por el Ministerio de Cultura, la Asociación de Gestión de

Gráfico 3 - España: formatos de publicación de las discográficas independientes, 2011

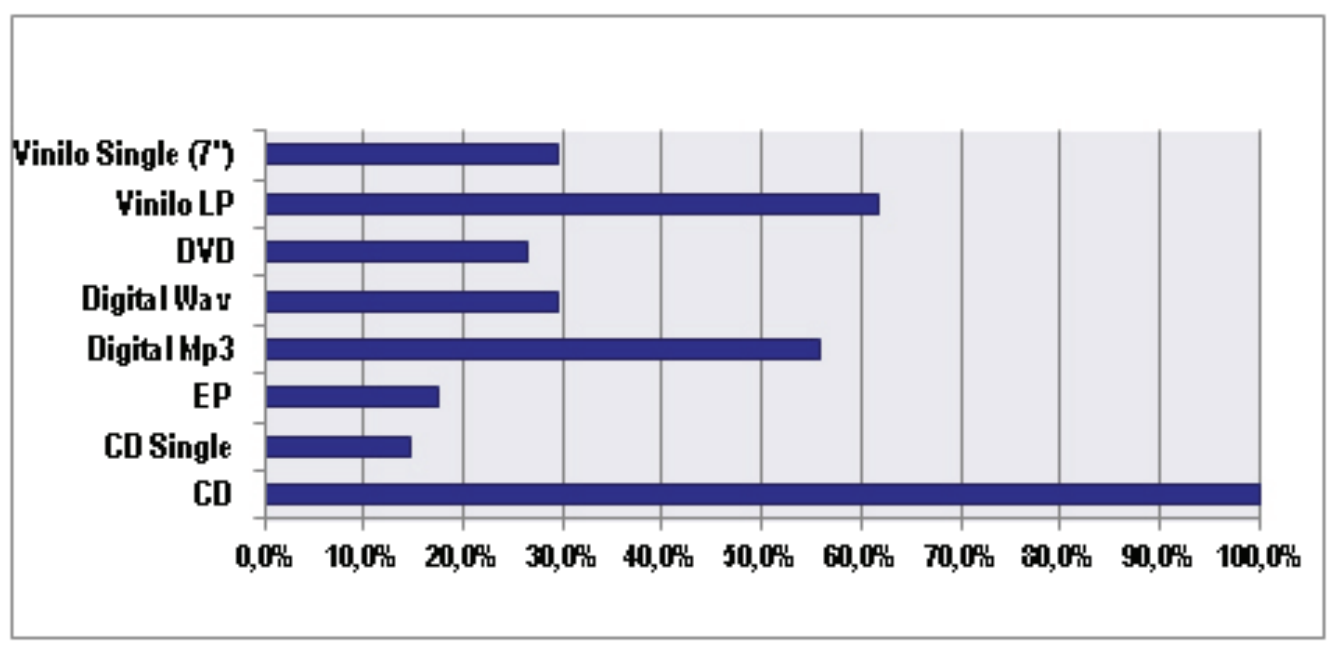

Fuente: elaboración propia. 
Derechos Intelectuales (AGEDI) y el Institut Ramón Llull a cargo de la promoción de la lengua catalana.

\section{Promoción y publicidad}

Otro aspecto que abarcó el estudio fue el de los principales medios publicitarios y actividades de promoción que encaran las compañías independientes. Una de las sorpresas reveladas es el hecho de que la cartelería en vía pública continúa siendo el principal medio en el cual las compañías invierten parte de sus presupuestos (80\% de 25 respuestas obtenidas). En la mayoría de los casos, al dinero destinado a este soporte publicitario se suman recursos para la impresión y distribución de flyers en mano y en circuitos de salas de conciertos, tiendas y bares, y la inclusión de publicidad en internet (52\%, respectivamente). Todos estos canales de contacto con los potenciales consumidores no implican grandes inversiones y garantizan cierto grado de efectividad. Contrariamente, la inversión de los sellos independientes en anuncios en prensa internacional, en blogs musicales o en el medio radiofónico es muy baja.

En relación con las actividades de promoción que complementan la inversión publicitaria, se destaca que la totalidad de las sellos (sobre 33 respuestas recibidas) incurre en la práctica enviar CD a los medios de comunicación; emisoras de radio y revistas especializadas, principalmente. En segundo término, utilizan profusamente (97\%) las listas de correo electrónico para mantener informados a sus potenciales clientes sobre las novedades musicales y graban videoclips de canciones de sus artistas (91\%). Adicionalmente la mayoría de las discográficas organiza conciertos gratuitos (64\% aprox.).

Gráfico 4 - España: inversión en publicidad de los sellos y distribuidoras independientes, 2011

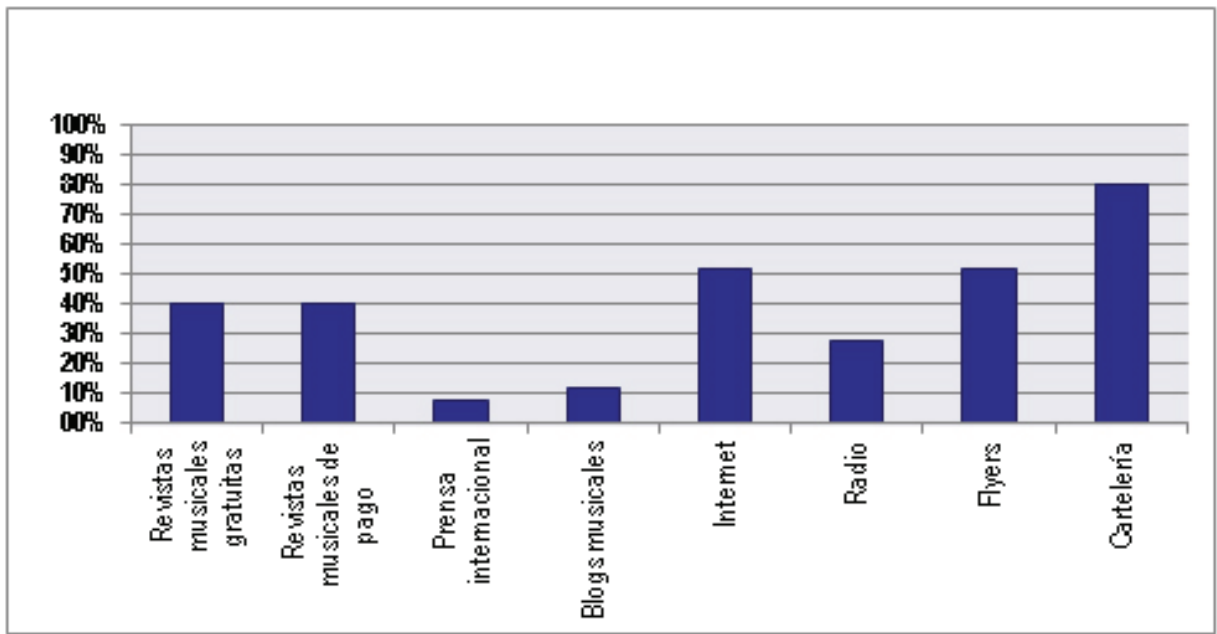

Fuente: elaboración propia. 
Por otra parte, la encuesta contenía una pregunta abierta acerca de si los sellos conocían la forma en que llegan a contactar con sus clientes. Un 61\% (sobre 31 respuestas recibidas) afirmó que sí. La mayoría de las respuestas obtenidas hizo referencia a la importancia de amigos y conocidos como prescriptores musicales, seguidos, por este orden, por las acciones de promoción realizadas en los conciertos, la presencia en las redes sociales digitales (Facebook, MySpace, YouTube...), las referencias en los medios de comunicación tradicionales y la presencia en internet.

\section{Internet y servicios online}

Respecto a la presencia de los sellos independientes en las diferentes redes sociales digitales y de sus repertorios en los servicios musicales de distribución, cabe destacar que la casi totalidad de las empresas han desarrollado sus propios sitios web y tienen una cuenta en Facebook (97\% y 93\% sobre 31 respuestas obtenidas, respectivamente). Esta plataforma sobre la que terceros pueden desarrollar aplicaciones y hacer negocios, se ha convertido en el referente fundamental de cara a promocionar artistas y trabajos musicales, y entablar relaciones con clientes y fans. Esta importancia se ve reflejada en que algunas de las empresas (26\%) tienen su propia aplicación en la red social digital.

A cierta distancia de Facebook se posicionan Myspace (71\%) y Youtube (68\%) como las siguientes redes con mayor presencia entre las independientes españolas. La primera de éstas ha sufrido un desmoronamiento general de usuarios pasando de ser un medio de difusión clave a jugar un papel secundario. Su decadencia puede atribuirse al surgimiento de nuevas plataformas de distribución de música online, como Spotify, Bandcamp o SoundCloud. Por su parte, el canal de

Gráfico 5 - España: actividades de promoción de los sellos independientes, 2011

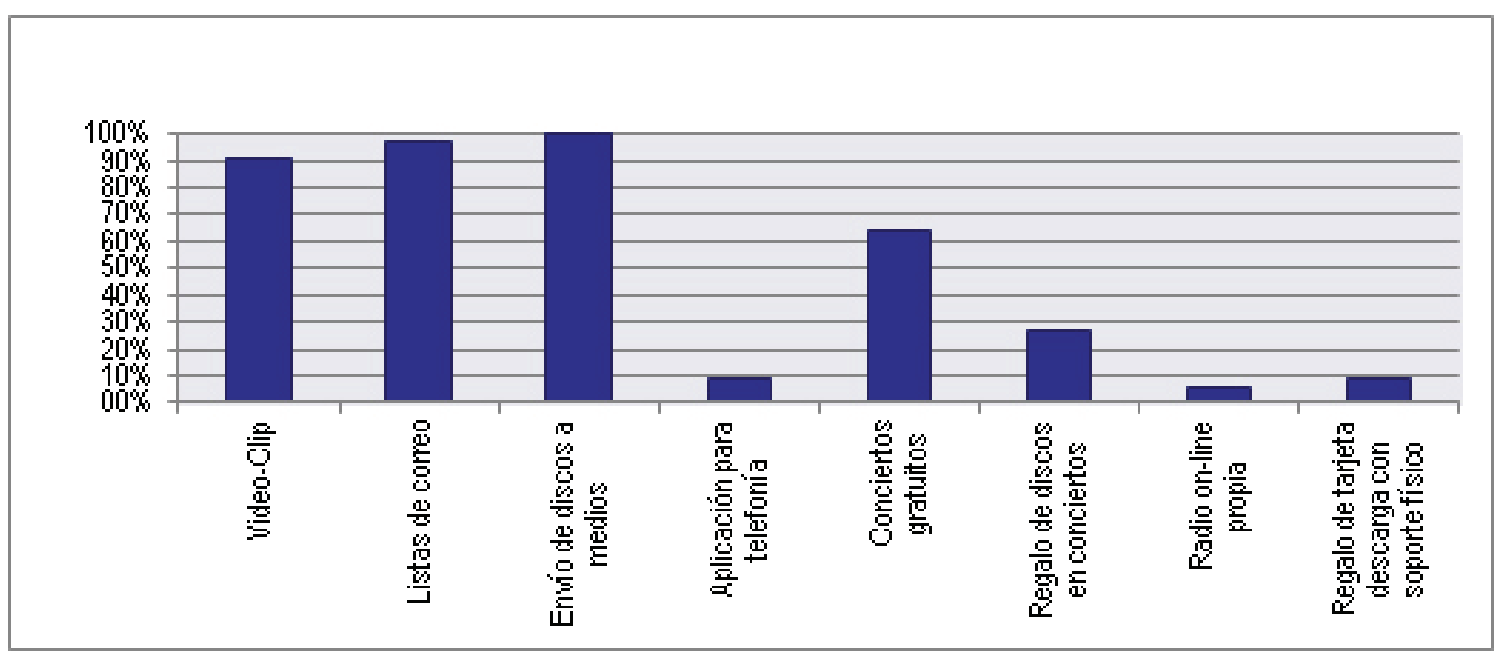

Fuente: elaboración propia. 
videos YouTube $u$ otras redes como Vimeo ganan peso cada día a la hora de difundir video-clips 0 actuaciones en vivo de diferentes músicos.

Por otra parte, Twitter también empieza a cobrar importancia como canal de noticias e información. Un 60\% (sobre 31 respuestas recibidas) de los sellos consultados posee su propio perfil. Por el contrario, llama la atención la falta de presencia de las compañías independientes en la red social española Tuenti (10\%). Esta plataforma, con cerca de 13 millones de usuarios (febrero de 2012), alberga un perfil de usuario adolescente, clave para muchos sellos independientes del país.

Actualmente hay una proliferación de servicios musicales digitales, especialmente en el entorno europeo donde se calcula que existen 465 servicios online (MULLIGAN, 2011). Desde el punto de vista de los modelos de comercialización que estos nuevos servicios de distribución sonora, nacionales e internacionales, se puede trazar la siguiente tipología: a) por suscripción, en streaming (caso Spotify Premium) o descarga; b) gratuitos financiados con publicidad (caso LastFM, Deezer o Rockola), c) y otros, como el pago por descarga y la descarga de contenidos para teléfonos móviles (AMETIC, 2011, p. 49-50).

El actual dominio de la aplicación para reproducción de música vía streaming Spotify en el mercado de música digital español es clave. Casi la totalidad de las empresas independientes (94\% sobre 34 respuestas obtenidas) tienen presencia en la plataforma de origen sueco. Si fijamos nuestra atención en el resto de los servicios musicales, sólo la presencia de las independientes supera el 50\% en el caso de Last.Fm.

Gráfico 6 - España: presencia en internet y redes digitales de los sellos independientes, 2011

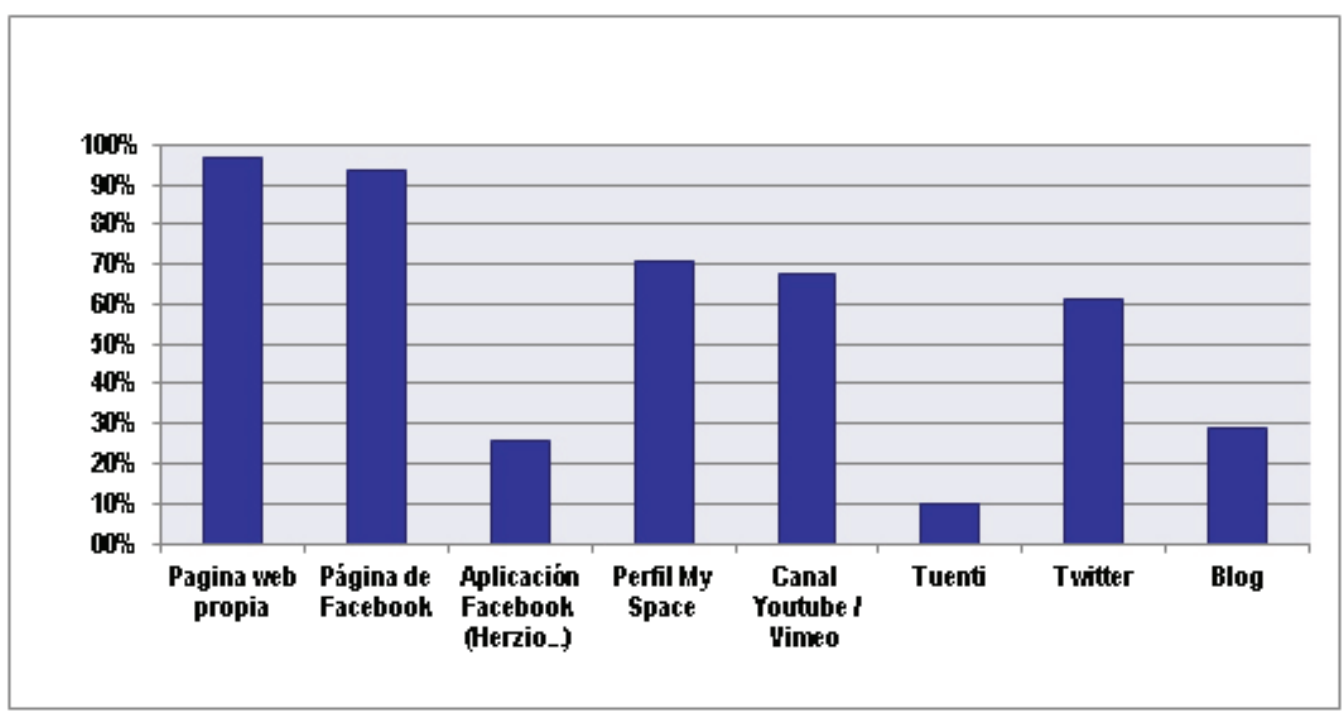

Fuente: elaboración propia. 
La presencia de obras en este tipo de servicios está mediada por la figura de los denominados "agregadores" o distribuidoras digitales, empresas que ofrecen sus servicios a sellos y artistas para disponibilizar catálogos y obras musicales en plataformas y tiendas online y en dispositivos móviles en diferentes mercados. Entre los agregadores utilizados por los sellos independientes encuestados se destacan principalmente la estadounidense The Orchard y la española Altafonte. Casi el $80 \%$ de los sellos consultados (sobre 34 respuestas recibidas) cede los derechos de comercialización de su catálogo a un agregador; un 47\% lo hace de forma exclusiva y un $32 \%$ de forma parcial. Mientras que sólo una minoría de empresas (23\%) gestiona directamente la distribución digital de su catálogo.

\section{Conclusiones}

En primer término, como apuntamos en otro trabajo (ALBORNOZ; GALLEGO, 2011), debe señalarse la opacidad en que se encuentra inmerso el sector de empresas independientes que actúan en la música popular española. Es flagrante la falta de estudios sistemáticos que den cuenta del peso económico y de las características de un sector conformado por un gran número de empresas diseminadas en la geografía española. Por otro lado, sería importante contrastar el volumen de artistas noveles que aportan al mercado así como evaluar su impacto en otras industrias culturales con las que se relacionan (bandas sonoras de películas, videojuegos, series y publicidad, presencia real en radios, inversión total en publicidad en medios escritos...). Por

Gráfico 7 - España: presencia de los sellos independientes en los servicios online, 2011

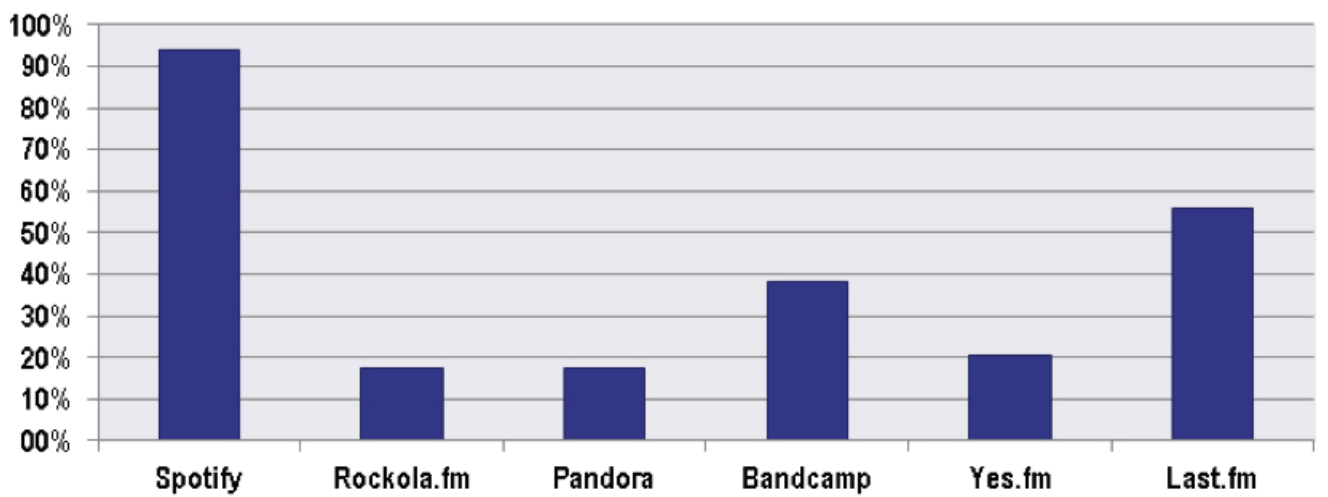


último, si bien uno de los objetivos específicos del estudio era conocer la facturación total del sector con la idea de calcular el impacto de cada una de las actividades, esta ha sido imposible de lograr por la baja respuesta a ese tipo de cuestiones.

Una segunda observación tiene relación con las diferentes y variadas funciones que hoy desarrollan las micro y pequeñas empresas que conforman este sector. En un escenario convulso, caracterizado por un mercado analógico en caída libre y uno digital que enfrenta dificultades a la hora de monetizar sus productos y servicios, estas firmas no sólo continúan dedicándose a la producción de fonogramas en una variedad de formatos (CD, discos de vinilo, archivos Mp3...), sino que también se han volcado a la contratación de artistas y bandas musicales nacionales (y en algunos casos extranjeras) y a prestar servicios de editorial. La contratación de músicos vincula a estas firmas con el sector de la música en vivo y sus agentes (agencias multinacionales de booking, promotores musicales locales, técnicos de sonido, gestores de espacios culturales, etc.), mientras que a través de los servicios que prestan en calidad de editoras velan por la explotación comercial de la obra de sus artistas en otras industrias culturales (cine, radio, televisión, publicidad, etc.). En resumen, las divisiones por tipo de actividad desarrollada que caracterizaban al sector hace algunos años se han desdibujado completamente, hoy en día los sellos y las distribuidoras independientes se han transformado en empresas de (múltiples) servicios musicales.
Cabe reseñar, en tercer lugar, la diversidad de estilos de la denominada música popular con la que trabajan los sellos y las distribuidoras fonográficas de este sector. Si bien la mayoría de los sellos trabaja con Pop y/o Rock, son pocas las firmas especializadas en un género particular; por el contrario, abundan las compañías que trabajan simultáneamente con varios estilos musicales. La estrategia de contar con catálogos estilísticamente diversificados es una respuesta de las independientes ante un escenario de fuerte competencia y presupuestos ajustados. Por otra parte, es fundamental el aporte de sellos y distribuidoras en calidad de importadoras y de exportadoras de obras musicales, ya sea licenciando/comprando licencias o distribuyendo materiales de diverso origen. La exportación de músicas autóctonas hacia otros mercados se viene potenciando en los últimos años con la presencia de empresas independientes locales en diversas ferias internacionales y festivales de promoción.

En cuarto lugar, pese a la fuerte caída de las ventas de música grabada en soporte CD y el crecimiento paulatino del mercado digital, que en 2011 representó en España un 31\% del mercado total, el CD continúa siendo el soporte empleado por la totalidad de las empresas que respondieron la encuesta online. La tangibilidad del CD hace que, por el momento, este continúe siendo considerado un medio idóneo para promocionar artistas y bandas entre los medios de comunicación tradicionales y nuevos. 
Por último, el estudio revela la combinación de diferentes tipos de actividades publicitarias y de promoción que encaran las empresas independientes. Al respecto, resulta evidente la coexistencia de un savoir faire tradicional, asentado en las inversiones en publicidad callejera, flyers y el envío de materiales a medios especializados, y de un escenario digital que pone a disposición de las empresas una serie de herramientas novedosas. En este sentido, las independientes han abrazado las posibilidades que brinda internet no sólo elaborando sus propios sitios web, que incluyen tiendas online, sino con una activa presencia en plataformas como Facebook, YouTube, Myspace o Twiter y, a través de los agregadores digitales, en servicios musicales como Spotify y Last.fm.

\section{Referencias}

ALBORNOZ, Luis A. La industria de la música. Un escenario en plena transformación. In BUSTAMANTE, Enrique (Coord.). Las industrias culturales audiovisuales e internet: experiencias, escenarios de futuro y potencialidades desde la periferia. La LagunaTenerife: IDEC0, 2011a. P. 47-66.

. La industria de la música en España: una actualidad compleja y crítica. In BUSTAMANTE, Enrique (Coord.). Informe sobre la cultura española y su proyección global, 2011. Madrid: Fundación Alternativas: Marcial Pons, 2011b. p. 67-87.

ALBORNOZ, Luis A.; GALLEG0, J. Ignacio. Setor da música independente?... Apontamentos sobre a trama empresarial espanhola. In: HERSCHMAN, Micael (Org.). Nas bordas e fora do mainstream musical: novas tendências da música independente no início do século XXI. São Paulo: Estação das Letras e Cores
Editora: FAPERJ, 2011. p. 87-104.

AMETIC - Asociación de Empresas del Sector TICC, las Comunicaciones y los Contenidos digitales. Informe de la industria de contenidos digitales 2011. Madrid: AMETIC, 2011.

BUQUET, Gustavo. La industria discográfica: reflejo tardío y dependencia del mercado internacional. In BUSTAMANTE, Enrique (Coord.). Comunicación y cultura en la era digital: industrias, mercados y diversidad en España. Madrid: Gedisa, 2002. p. 67-105.

FONAROW, Wendy. Empire of Dirty: the Aesthetics and Rituals of British Indie Music. Middleton (Connecticut): Wesleyan University Press, 2006.

GALLEGO, J. Ignacio. Novas formas de prescrição musical. In: HERSCHMANN, Micael (Org.). Nas bordas e fora do mainstream musical: novas tendências da música independente no início do século XXI. São Paulo: Estação das Letras e Cores Editora: FAPERJ, 2011. p. 47-59.

HESMONDHALGH, David. Indie: The institutional politics and aesthetics of a popular music genre. Cultural Studies, v. 13, n. 1, p. 34-61, 1999.

KRUSE, Holly. Site and Sound: Understanding Independent Music Scenes. New York: Peter Lang, 2003.

MULLIGAN, Mark. Is digital music the Next Eurozone CrisisWaiting to Happen? Music Industry Blog. Nov. 2011. Disponible en: < http://musicindustryblog.wordpress. com/2011/11/29/is-digital-music-the-next-eurozone-crisiswaiting-to-happen >. Acceso: 10 feb. 2012.

PROMUSICAE - Productores de Música de España. Participación de compañías en el mercado discográfico físico, año 2011. Madrid: PROMUSICAE, 2012a. Disponible en: < http://www.promusicae.es/ espanol.html>. Acceso: 13 dic. 2011.

. Participación de compañías en el mercado discográfico digital, año 2011. Madrid: PROMUSICAE, 2012b. Disponible en: < http://www.promusicae.es/ 
espanol.html>. Acceso: 13 dic. 2011.

SGAE - Sociedad General de Autores y Editores.

Anuario SGAE de las Artes Escénicas, Musicales y

Audiovisuales 2012. Madrid: SGAE, 2011.

SHUKER, Roy. Diccionario del rock y de la música

popular. Barcelona: Ma Non Troppo (Ediciones

Robinbook), 2005. [Original en inglés: Popular Music.

The Key Concepts. Londres: Routledge].

UFI - Unión Fonográfica Independiente. Libro blanco. Madrid: UFI, 2003.

¿Qué es UFI? UFIMusica.com. Madrid: UFI.

Disponible en: < http://www.ufimusica.com/plantillas/ que_es.php?newlang=es >.Acceso: 13 dic. 2011. 
ANEXO A - Sellos discográficos y distribuidoras musicales participantes en el estudio Situación de la industria musical independiente en España, 2011.

\begin{tabular}{|c|c|c|c|}
\hline & Sello & Localización & Página Web \\
\hline 1. & 18 Chulos Records & Madrid & 18chulos.com \\
\hline 2. & Absolute Beginners & Madrid & absolutebeginners.es \\
\hline 3. & B0A Música & Madrid & boamusica.com \\
\hline 4. & Cigala Music & Madrid & elcigala.com/cigalamusic \\
\hline 5. & Discmedi Blau & Barcelona & discmedi.com \\
\hline 6. & Acuarela Discos & Madrid & acuareladiscos.com \\
\hline 7. & El Genio Equivocado & Barcelona & elgenioequivocado.com \\
\hline 8. & El Volcán Música & Madrid & elvolcanmusica.com \\
\hline 9 & Elefant Records & Madrid & elefant.com \\
\hline 10. & Ernie Producciones & Pontecaldelas (Pontevedra) & ernieproducciones.com \\
\hline 11. & Esmerarte Servicios Artísticos & Vigo & esmerarte.com \\
\hline 12. & Everlasting Records / Pop Stock & Madrid & everlastingrecords.com \\
\hline 13. & Federación de Universos Pop & Madrid & federacionuniversospop.com \\
\hline 14. & Foehn Records & Barcelona & foehnrecords.com \\
\hline 15. & Gaztelupeko Hotsak & Soraluze (Guipúzcoa) & hotsak.com \\
\hline 16. & Globomedia Música & Madrid & globomedia.es/artistas.aspx \\
\hline 17. & Gramaciones Grabofónicas & Madrid & gramacionesgrabofonicas.com \\
\hline 18. & Gran Derby Records & Madrid & granderbyrecords.com \\
\hline 19. & I'm An Artist & Madrid & imanartist.es \\
\hline 20. & Jabalina Música & Madrid & jabalinamusica.com \\
\hline 21. & Kankana Records & Malaga & \\
\hline 22. & La Ecléctica Madrileña & Madrid & laeclecticamadrilena.es \\
\hline 23. & La Huella Sonora & Madrid & lahuellasonora.com \\
\hline 24. & Limbo Starr & Madrid & limbostarr.com \\
\hline 25. & Lola Bourne Management \& Music & Madrid & lolabournemusic.com \\
\hline 26. & Lonxa Cultural & A Coruña & lonxacultural.com \\
\hline 27. & Lovemonk & Madrid & lovemonk.net \\
\hline 28 & Mús Records & Burgos & musrecords.com \\
\hline 29. & Mushroom Pillow Music & Madrid & mushroompillow.com \\
\hline 30. & I+D Music & Palma de Mallorca & imasdemusic.com \\
\hline 31. & Naïve Discos España & Barcelona & naive.es \\
\hline 32. & Nesma Music & Madrid & nesma.es \\
\hline 33. & Ojo Música & Galapagar (Madrid) & galileo-mc.com \\
\hline 34. & Origami Records & Madrid & origamirecords.com \\
\hline 35. & Pias Spain & Madrid & pias.com/es \\
\hline 36. & Primeros Pasitos & Palma de Mallorca & primerospasitos.com \\
\hline 37. & Producciones Doradas & Barcelona & produccionesdoradas.com \\
\hline 38. & Resistencia & Madrid & resistencia.es \\
\hline 39. & Sones & Barcelona & sones.es \\
\hline 40. & Starsky Records & Madrid & \\
\hline 41. & Wild Punk Records & Granada & wildpunk.com \\
\hline
\end{tabular}


The popular music industry in Spain: independent record labels in the digital era

\section{Abstract}

This article introduces the main results of a survey performed on independent record and distribution companies of popular music in Spain. The goal of this research was to obtain a sort of portrait of this sector and to analyze its present tendencies. An online survey was conducted during the first semester of 2011 by researchers of the Journalism and Media Studies Department at Carlos III University of Madrid, with the support of the Spanish Association and Independent Phonographic Union, best known by the acronym UFI. The article emphasizes mainly the different activities and strategies developed by independent record labels and distribution companies. These companies show a very active presence on the new digital networks and platform services. The results of the research are introduced in the following order: a) profile of the companies, b) international presence, c) advertisement and promotional strategies, and d) internet and online services.

\section{Keywords}

Music Industry. Independent Record Companies. Internet. Popular Music. Spain.

\section{A indústria da música popular na Espanha: os selos independentes na era digital}

\section{Resumo}

0 artigo apresenta os resultados de um estudo realizado com gravadoras e distribuidoras independentes que atuam no mercado de música popular na Espanha. Com a finalidade de obter uma espécie de fotografia da situação deste setor e analisar suas tendências, aplicamos um questionário online durante 0 primeiro semestre de 2011 a esse tipo de empresas. 0 estudo, dirigido por pesquisadores do Departamento de Jornalismo e Comunicação Audiovisual da Universidade Carlos III de Madri, contou com 0 apoio da União Fonográfica Independente. 0 artigo trata, principalmente, das diversas atividades e estratégias desenvolvidas pelos selos e distribuidoras independentes, que incluem uma ativa presença nas novas redes e plataformas digitais. Os resultados da pesquisa são apresentados na seguinte ordem: a) perfil das empresas, b) presença internacional, c) promoção e publicidade, e d) internet e serviços online.

\section{Palavras-chave}

Indústria musical. Gravadoras independentes. Internet. Música popular. Espanha. 


\section{Expediente}

A revista E-Compós é a publicação científica em formato eletrônico da Associação Nacional dos Programas de Pós-Graduação em Comunicação (Compós). Lançada em 2004, tem como principal finalidade difundir a produção acadêmica de pesquisadores da área de Comunicação, inseridos em instituições do Brasil e do exterior.

\section{E-COMPÓS I www.e-compos.org.br I E-ISSN 1808-2599}

Revista da Associação Nacional dos Programas

de Pós-Graduação em Comunicação.

Brasília, v.15, n.2, maio/ago. 2012.

A identificação das edições, a partir de 2008 ,

passa a ser volume anual com três números.

\section{CONSELHO EDITORIAL}

Afonso Albuquerque, Universidade Federal Fluminense, Brasil Alberto Carlos Augusto Klein, Universidade Estadual de Londrina, Brasil Álvaro Larangeira, Universidade Tuiuti do Paraná, Brasi André Luiz Martins Lemos, Universidade Federal da Bahia, Brasil Ângela Freire Prysthon, Universidade Federal de Pernambuco, Brasil Angela Cristina Salgueiro Marques, Faculdade Cásper Líbero (São Paulo), Brasil Antonio Roberto Chiachiri Filho, Faculdade Cásper Líbero, Brasil Arthur Autran Franco de Sá Neto, Universidade Federal de São Carlos, Brasil Benjamim Picado, Universidade Federal Fluminense, Brasil César Geraldo Guimarães, Universidade Federal de Minas Gerais, Brasil Cristiane Freitas Gutfreind, Pontifícia Universidade Católica do Rio Grande do Sul, Brasil

Denilson Lopes, Universidade Federal do Rio de Janeiro, Brasil Eduardo Peñuela Cañizal, Universidade Paulista, Brasi Eduardo Vicente, Universidade de São Paulo, Brasil Eneus Trindade, Universidade de São Paulo, Brasil Florence Dravet, Universidade Católica de Brasília, Brasil Gelson Santana, Universidade Anhembi/Morumbi, Brasil Gislene da Silva, Universidade Federal de Santa Catarina, Brasil Guillermo Orozco Gómez, Universidad de Guadalajara Gustavo Daudt Fischer, Universidade do Vale do Rio dos Sinos, Brasil Hector Ospina, Universidad de Manizales, Colômbia Herom Vargas, Universidade Municipal de São Caetano do Sul, Brasil Inês Vitorino, Universidade Federal do Ceará, Brasil Jay David Bolter, Georgia Institute of Technology Jeder Silveira Janotti Junior, Universidade Federal de Pernambuco, Brasil John DH Downing, University of Texas at Austin, Estados Unidos José Afonso da Silva Junior, Universidade Federal de Pernambuco, Brasil José Carlos Rodrigues, Pontifícia Universidade Católica do Rio de Janeiro, Brasi José Luiz Aidar Prado, Pontifícia Universidade Católica de São Paulo, Brasil Kelly Cristina de Souza Prudêncio, Universidade Federal do Paraná, Brasil. Laan Mendes Barros, Universidade Metodista de São Paulo, Brasil
Lance Strate, Fordham University, USA, Estados Unidos

Lorraine Leu, University of Bristol, Grã-Bretanha

Lucia Leão, Pontifícia Universidade Católica de São Paulo, Brasil

Malena Segura Contrera, Universidade Paulista, Brasil

Márcio de Vasconcellos Serelle, Pontifícia Universidade Católica de Minas Gerais, Brasil

Maria Aparecida Baccega, Universidade de São Paulo e Escola Superior de Propaganda e Marketing, Brasil

Maria Ataide Malcher, Universidade Federal do Pará, Brasi

Maria das Graças Pinto Coelho, Universidade Federal do Rio Grande do Norte, Brasil

Maria Immacolata Vassallo de Lopes, Universidade de São Paulo, Brasil Maria Luiza Martins de Mendonça, Universidade Federal de Goiás, Brasi Mauro de Souza Ventura, Universidade Estadual Paulista, Brasil Mauro Pereira Porto, Tulane University, Estados Unidos Mirna Feitoza Pereira, Universidade Federal do Amazonas, Brasil Nilda Aparecida Jacks, Universidade Federal do Rio Grande do Sul, Brasil Osvando J. de Morais, Universidade de Sorocaba, Brasil Potiguara Mendes Silveira Jr, Universidade Federal de Juiz de Fora, Brasil Renato Cordeiro Gomes, Pontifícia Universidade Católica do Rio de Janeiro, Brasil Robert K Logan, University of Toronto, Canadá

Ronaldo George Helal, Universidade do Estado do Rio de Janeiro, Brasil Rose Melo Rocha, Escola Superior de Propaganda e Marketing, Brasil Rossana Reguillo, Instituto de Estudos Superiores do Ocidente, Mexico Rousiley Celi Moreira Maia, Universidade Federal de Minas Gerais, Brasil Sebastião Guilherme Albano da Costa, Universidade Federal do Rio Grande do Norte, Brasil

Simone Maria Andrade Pereira de Sá, Universidade Federal Fluminense, Brasil Tiago Quiroga Fausto Neto, Universidade de Brasília, Brasil Suzete Venturelli, Universidade de Brasília, Brasil Valerio Fuenzalida Fernández, Puc-Chile, Chile Veneza Mayora Ronsini, Universidade Federal de Santa Maria, Brasil Vera Regina Veiga França, Universidade Federal de Minas Gerais, Brasil

\section{COMISSÃO EDITORIAL}

Adriana Braga I Pontifícia Universidade Católica do Rio de Janeiro, Brasil

Felipe Costa Trotta I Universidade Federal Fluminense, Brasi

CONSULTORES AD HOC

Adriana Amaral, Universidade do Vale do Rio dos Sinos, Brasil

Ana Carolina Escosteguy, Pontifícia Universidade Católica do Rio Grande do Sul, Brasi Claudia Azevedo, Universidade Federal do Estado do Rio de Janeiro, Brasil Gisela Castro, Escola Superior de Propaganda e Marketing, Brasil Luis Queiroz, Universidade Federal da Paraíba, Brasil Rodrigo Carreiro, Universidade Federal de Pernambuco, Brasil EDIÇÃO DE TEXTO E RESUMOS I Susane Barros SECRETÁRIA EXECUTIVA I Juliana Depiné EDITORAÇÃO ELETRÔNICA I Roka Estúdio TRADUÇÃo I Sieni Campos
COMPÓS I www.compos.org.br

Associação Nacional dos Programas de Pós-Graduação em Comunicação

Presidente

Julio Pinto

Pontifícia Universidade Católica de Minas Gerais, Brasil juliopinto@pucminas.br

Vice-presidente

Itania Maria Mota Gomes

Universidade Federal da Bahia, Brasil

itania@ufba.br

Secretária-Geral

Inês Vitorino

Universidade Federal do Ceará, Brasil

inesvic@gmail.com 